\title{
Study on the Free-positioning of ETS in the PDL Track Surveying System
}

\author{
Hongtao Zhu, Weijun Wu \& Zhiyong Wang \\ School of Mechanical and Electrical Engineering \\ Nanchang University \\ Nanchang 330031, China \\ E-mail:wwjott@163.com
}

\begin{abstract}
The station positioning by the electric total station (ETS) is the first step which must be done for the passenger dedicated line track survey and one of the important factors which influence the final surveying precision. To enhance the surveying efficiency and surveying precision, we study the principles and methods of free positioning by ETS.
\end{abstract}

Keywords: Passenger dedicated line (PDL), Electronic total station (ETS), Free-station positioning

\section{Introduction}

The PDL track surveying system adopts ETS to survey the 3D coordinate of the track, and the positioning of ETS is the necessary work before the normal survey, and the resection method is generally adopted. This positioning method doesn't require the position of the ETS which can be freely set on one side of the track or the centre of the track, so it is also called the free-positioning method. When freely positioning, ETS will observe the prism on the control foundation pile point (CPIII) with compulsory centering device, and we can obtain the side length and the angle between the ETS and prism point. According to the principle of the free positioning, we only need survey two foundation pile points to confirm the station coordination and back sight direction of ETS. But according to the precision requirements of the permanent CPIII control net (the relative precision of the plane is $\pm 1 \mathrm{~mm}$, and the relative precision of the altitude is $\pm 0.5 \mathrm{~mm}$ ) (Chinese Ministry of Railway, 2006), comprehensively considering the surveying precision index of ETS $\left(0.5^{\prime \prime}, 1+1 \mathrm{ppm}\right)($ Fan, 2003), the surveying uncertainty can not fulfill the position precision of the track 3D coordinate surveying (the precision usually should be controlled in $\pm 1.0 \mathrm{~mm}$ ) only through observing two foundation pile points. So in the actual project, we usually adopt the surplus surveying (such as $8 \sim 12$ points), the set collection surveying and other measures to enhance the positioning precision through error compensation. The cost is to add the surveying workload and reduce the efficiency of the positioning. The research of the free positioning of ETS is to utilize the auto-collimating function of ETS, control the autorotation, object searching, auto-surveying of ETS by the software, and realize the high-efficient and precise auto-survey of ETS to the surplus observation and set collection without human operation.

\section{Basic principles of ETS for free positioning}

When freely positioning, after ETS levels off and achieves stable, a self surveying coordinate system has existed in ETS, but this coordinate system is not relative with the coordinate system which the control foundation pile points are located. So the precondition of free positioning is to implement cursory positioning by manual surveying and obtain the station coordinates of ETS in the control foundation pile net coordinate system and the selected horizontal reference angle $A h_{0}$, and establish the conversion relationship between two coordinate systems and settle bases for the autorotation and collimation and survey of ETS controlled by the software.

The resection computation formulas of the station coordinates of ETS are

$$
\begin{aligned}
& x_{0}=x_{1}+\left(x_{2}-x_{1}\right) \times L+\left(y_{2}-y_{1}\right) \times H \\
& y_{0}=y_{1}+\left(y_{2}-y_{1}\right) \times L+\left(x_{2}-x_{1}\right) \times H \\
& z_{0}=\frac{\left(z_{1}^{\prime}-z_{1}+z_{2}^{\prime}-z_{2}\right)}{2} \\
& A h_{0}=\arctan \left(\frac{y_{1}^{\prime}-y_{0}}{x_{1}^{\prime}-x_{0}}\right)
\end{aligned}
$$


where, $L=\frac{\left(s_{1}{ }^{2}+s_{0}{ }^{2}-s_{2}{ }^{2}\right)}{2 \times s_{0}{ }^{2}}, \quad H=\sqrt{s_{1}^{2} /\left(s_{0}{ }^{2}-L^{2}\right)}, \quad s_{0}=\sqrt{\left(x_{1}-x_{2}\right)^{2}+\left(y_{1}-y_{2}\right)^{2}}, \quad s_{1}=\sqrt{x_{1}^{2}+y_{1}^{2}}, \quad s_{2}=\sqrt{x_{2}^{2}+y_{2}^{2}}$,

$\left(x_{1}^{\prime}, y_{1}^{\prime}, z_{1}^{\prime}\right)$ and $\left(x_{2}^{\prime}, y_{2}^{\prime}, z_{2}^{\prime}\right)$ respectively are the surveying coordinates of the foundation pile point 1 and the foundation pile point 2 under the coordinate system of ETS, $\left(\mathrm{x}_{1}, \mathrm{y}_{1}, \mathrm{z}_{1}\right)$ and $\left(\mathrm{x}_{2}, \mathrm{y}_{2}, \mathrm{z}_{2}\right)$ respectively are the appointed coordinates of the foundation pile point 1 and the foundation pile point 2 under the geodetic coordinate system, and ( $\mathrm{x}_{0}$, $\left.\mathrm{y}_{0}, \mathrm{z}_{0}\right)$ is the station coordinate computed by the resection method.

The requirement of free positioning is that ETS implements automatic surveying for the surplus surveys and set collection surveys and obtains the station coordinates with high precision through strict error compensation. And its basic principle induces four aspects.

(1) The route layout of free positioning

ETS adopts the mode of P2P to survey the appointed foundation pile points when freely positioning, and its route can rotate according to certain direction or selected sequence. The precondition quickly turning from one appointed surveying point to the next surveying point is that the position of the surveyed point can be confirmed. So, the free positioning software picks up the $3 \mathrm{D}$ coordinates under the geodetic coordinate system in the database according to the next surveyed foundation pile number, and confirms the horizontal angle and the vertical angle of the surveyed foundation pile prism point under the survey system of ETS through coordinate conversion.

Taking the computation of the horizontal angle of the $i$ 'th prism point as the example, through the coordinates of the stations of ETS, the selected back sight datum mark and the searched foundation pile point, we can obtain the horizontal deflection angle of the researched point relative to the back sight datum mark $A h_{\mathrm{i}}$.

$A h_{i}=\arctan \left(\frac{y_{i}-y_{0}}{x_{i}-x_{0}}\right)-\arctan \left(\frac{y_{1}-y_{0}}{x_{1}-x_{0}}\right)\left\{0 \leq A h_{i} \leq 2 \times \pi\right\}$

$A h_{\mathrm{i}}+A h_{0}$ is the horizontal angle of the $i$ 'th prism point in the coordinate system of ETS.

The computation method of the vertical angle is simple, and it needs not to compute the benchmark angle like the horizontal angle because it only needs to select the $0^{0}$ of the zenith angle as the vertical benchmark angle, so the vertical angle $A v_{i}$ is

$A v_{i}=\arctan \left(\frac{\sqrt{\left(x_{i}-x_{0}\right)^{2}+\left(y_{i}-y_{0}\right)^{2}}}{z_{i}-z_{0}}\left\{0 \leq A v_{i} \leq 2 \times \pi\right\}\right.$

After confirming the horizontal rotation angle $A h_{\mathrm{i}}$ and the vertical rotation angle $A v_{i}$, we can find out the surveyed point.

(2) The implementation of the manual working of ETS

We can start the automatic search function of ETS to make ETS to collimate the surveyed point and automatically complete survey and data upload.

ETS turns to the surveyed point by the mode of P2P, and considering the station coordination of the primary position of ETS is not exact enough, and the errors exist between the actual position of the foundation pile prism point and its theoretical position, we should control ETS to quickly search the prism in a small appointed search range taking the direction as the centre through orders.

To enhance the nicety of the rotation and the efficiency of the search, after survey one foundation pile point, we should add the survey result into the surveyed data, and recomputed the station coordinate of ETS through error compensation to make the coordinate precision of the station gradually enhanced.

\section{(3) Set collection survey}

To eliminate or reduce the survey error of ETS direct telescope or reverse telescope, we need to adopt one set collection survey or multiple set collections survey. When computing the error compensation, we should take the average value of the direct telescope or reverse telescope of every set collection as the final survey data. And the data formats of different brand ETS may be different, for example, the once set collection average of Topcon ETS is the half of the difference between the direct telescope survey value and the reverse telescope survey value, and the once set collection average of Leica ETS is the half of the sum of the direct telescope survey value and the reverse telescope survey value.

(4) Computation of the station coordinates

According to the results of the surplus survey and multiple set collection survey of ETS, the error equation listed through the indirect error compensation method is (You, 1991)

$$
V_{s i}=\cos \alpha_{i} d x_{p}+\sin \alpha_{i} d y_{p}+l_{i}
$$




$$
\begin{aligned}
& V_{\gamma j}=\left(\frac{\rho}{S_{j}} \sin \alpha_{i}+\frac{\rho}{S_{j+1}} \sin \alpha_{i+1}\right) d x_{p}+\left(\frac{\rho}{S_{j}} \cos \alpha_{i}+\frac{\rho}{S_{j+1}} \cos \alpha_{j+1}\right) d y_{p}+\omega_{j} \\
& l_{i}=S_{i}^{0}-S_{i} \\
& \omega_{j}=\gamma_{j}^{0}-\gamma_{j} \\
& i=1,2 \cdots 8 \\
& j=1,2 \cdots 7
\end{aligned}
$$

Where, $\alpha_{\mathrm{i}}$ is the coordinate azimuth angle approximation from the point $i$ to $\mathrm{P}, S_{i}$ is the survey value of the $i$ 'th side, $S_{i}^{0}$ is the approximation of the $i$ 'th side, $\gamma_{\mathrm{j}}$ is the survey value of the included angle between the $j$ 'th side and $j+1$ 'th side, $\gamma_{j}^{0}$ is the approximation of the included angle between the $j$ 'th side and $j+1$ 'th side, $S_{i}^{0}$ and $\gamma_{j}^{0}$ can be obtained through the back calculation of the approximate coordinates of the station.

So we can obtain the matrix equations set, $V_{n \times 1}=\underset{n \times 1}{B} \cdot \delta \underset{t \times 1}{X}+l_{n \times 1} \quad(\mathrm{n}=15, \mathrm{t}=2)$, and it is the consistent equations which have two undetermined correction quantities and 15 unknowns, and there are 17 undetermined quantities and 15 equations, so the equations set has infinite groups of solution (Zhu, 2006).

$P=\left(\begin{array}{cccccc}P_{S 1} & 0 & & \ldots & & 0 \\ 0 & \ddots & & & & \\ & & P_{S 8} & & & \vdots \\ \vdots & & & P_{\gamma 1} & & \\ & & & & \ddots & 0 \\ 0 & & \ldots & & 0 & P_{\gamma 7}\end{array}\right)$

According to the free extremum of function, when $V^{T} P V=\min$, we can seek the unique solution. So we can obtain $B^{T} P V=0$, and to find the solution of the equation, we must confirm the matrix P. According to the survey principle of ETS (Fan, 2003), the surveying angles and the surveying sides are independent, so the weight matrix $\mathrm{P}$ is a diagonal matrix of $15 \times 15$, and $P_{S i}$ is the weight value of the surveying side.

Based on that, we can utilize the matrix inverse or the solution of Gauss equation to solute all unknown quantities such as $\mathrm{V}_{\mathrm{si}}, V_{\gamma i}, \mathrm{dx}$ and $\mathrm{dy}$. So we can obtain the final position coordinate.

$\left\{\begin{array}{l}x=x_{0}+d x \\ y=y_{0}+d y\end{array}\right.$.

\section{Intellectualization of ETS for free positioning}

The intellectualization of ETS for free positioning is mainly embodied in two aspects. First, it can make the system interface to be more humanism and adopt different operation habits, and further simplify the operation. Second, the system can timely reflect the abnormal instances in the process of free positioning and implement proper disposals or offer clues for the emergency disposal.

For the system interface and the function design, the convenience of operation and the perfection of the function are two important indexes of the system, and to avoid the complexity of the operation when perfecting the function is one of the important intellectualization directions of ETS for free positioning, and we should try to reduce the operation times and approaches, strengthen the aim and navigation of the operation, and make operators more relax.

At the same time, abnormal instances will occur in the free positioning unavoidably, and if the program has not preplans for the usual abnormal instances, it will influence the precision of the station, even make the positioning failed. To ensure free positioning of ETS with high quality and high efficiency, we have to efficiently capture and dispose these abnormal instances in time.

The abnormal instances which might occur in the free positioning include following instances at least.

(1) Prism identification

Because the track foundation pile net is set along both sides of the line, so when searching the selected station prism, 
other prisms might exist around the search sight line, and the wrong object always be found after searching and locking the prism. If the program could not judge this instance, though the positioning process can be completed, but the final residual error will largely exceed the anticipation, and the positioning will fail.

Another instance is that when manual surveying the former two foundation-pile points, if the actual surveying target doesn't match with the predetermined target (for example, the foundation-pile 1 and the foundation-pile 2 are selected, but the prism 1 and the prism 3 are surveyed), the prism search of ETS will fail when automatically turning to next foundation pile point and the positioning can not continue.

To solve these problems, the system should have the ability to identify the prism. ETS Trimble has a sort of special prism ID identification function, but many mainstream ETS such as Leica and Topcon have no that function. Therefore, we have to look for a sort of general solution. The simple method is to compute the difference between the theoretical position (horizontal angle, vertical angle and slop distance) after each prism survey is completed, and if the difference exceeds certain threshold value, so the prism doesn't match, we can search again or pause for the free positioning and disposal through the human-computer interaction.

(2) Bad sight line

When freely positioning, 8-12 prisms are distributed at both sides of the line of about $200 \mathrm{~m}$, and worker, vehicles and construction all might induce the ETS can not collimate or survey certain or some prisms, and the free positioning process will be interrupted or ceased.

When the sight line is bad, according to the feedback information from ETS, the free positioning software can clew the reason of error and the solution through the human-computer interaction, and allow operators survey again, skip or delete this point, and ensure the free positioning to go on wheels.

(3) Residual errors of positioning

Because of the loosening of individual foundation piles, the large error of the prism, the wrong database information of the foundation pile point, the instable tripod and quick changing temperature differences, the residual errors of positioning may exceed the limitation.

When the residual error of positioning exceeds the limitation, the free positioning software could clew that operators eliminate, survey again or permanently delete the data of the foundation pile point which exceeds the limitation.

\section{Conclusions}

The free positioning and the intellectualization of ETS can largely enhance the efficiency and the reliability of the positioning. The positioning process is mainly completed by the software, and few human-computer interactions are only operated on the remote computer, and the chance of the manual operation is reduced to the least, which can ensure the stability of the electric air bubble of ETS and various compensation values and make the surveying data more exact and reliable. At the same time, the decrease of the operation difficulty and the enhancement of the efficiency for the positioning can make the two-set-collection and multiple-set-collection possibly applied in the actual engineering.

The ETS free positioning in the PDL track surveying system has been successfully applied in the SGJ series of PDL track survey meter made by Jiangxi Everbright Industry Company, and the effect is very good.

\section{References}

Chinese Ministry of Railway. (2006). The Tentative Specifications on PDL Ballastless Track Railway Engineering Surveying. Beijing: China Railway Press.

Fan, Baixing. (2003). Processing Survey Data Automatically by Survey Robot. Beijing Surveying and Mapping. No.2. You, Zuji. (1991). Survey Adjustment and Tutorial. Beijing: Press of Surveying and Mapping. May of 1991.

Zhu, Baoxun, Liu, Chenglong \& Yang, Tianyu. (2006). Method of Resection and the Precision Estimation. Railway Investigation and Surveying. No.4. 